\title{
Actividad contra Trypanosoma cruzi (Kinetoplastida: Trypanosomatidae) de extractos metanólicos de plantas de uso medicinal en México
}

\author{
Karla C. Pérez, Lucio Galaviz, Jesús M. Iracheta, Eliud A. Lucero \& Zinnia J. Molina \\ Universidad Autónoma de Nuevo León, Laboratorio de Patología Molecular y Experimental, Facultad de Ciencias \\ Biológicas, Ave. Universidad SN, Ciudad Universitaria, C.P. 66455, San Nicolás de los Garza, Nuevo León, México; \\ aestusdrako@hotmail.com, lucio.galavizs1@uanl.edu.mx,jemairvi@gmail.com, \\ alonso_lucero_velasco@hotmail.com,molinazinnia@hotmail.com
}

Recibido 29-III-2017. Corregido 24-VII-2017. Aceptado 24-VIII-2017.

\begin{abstract}
Activity against Trypanosoma cruzi (Kinetoplastida: Trypanosomatidae) of methanolic extracts of medicinal use plants in Mexico. American trypanosomiasis is a potentially lethal disease caused by the hemoflagellate protozoan Trypanosoma cruzi. This neglected disease affects from 6 to 7 million people worldwide. Currently there are only two medicines to treat this disease: beznidazol and nifurtimox, both effective if they are administrated in the acute phase of infection, although their effectiveness fades away in the chronic phase; it also induces significant side effects. The aim of this study is to screen the trypanocidal activity of methanolic extracts from Hematoxilum brasiletto, Marrubium vulgare, Schinus molle, and Cympongon citratus, against $T$. cruzi epimastigotes, followed by the chromatographic separation, and identification of active compounds of the best candidate by colored chemical reactions; furthermore, it was also determined their cytotoxic effect in human lymphocytes and the brine shrimp Artemia salina. The extract of $H$. brasiletto showed the highest anti-T. cruzi activity with a inhibitory concentration $\left(\mathrm{IC}_{50}\right)$ of $543 \mu \mathrm{g} / \mathrm{mL}$; in descending order, it was followed by $M$. vulgare $\left(\mathrm{IC}_{50}=647 \mu \mathrm{g} / \mathrm{mL}\right), S$. molle $(\mathrm{IC}=827 \mu \mathrm{g} / \mathrm{mL})$ and finally, C. citratus $(\mathrm{IC}=1210$ $\mu \mathrm{g} / \mathrm{mL})$. The chromatographic fraction $\mathrm{Fr} 22$ from $H$. brasiletto showed the best anti- $T$. cruzi effectivity $\left(\mathrm{IC}_{50}=\right.$ $0.238 \mathrm{mg} / \mathrm{mL}$ ), when compared to the other fraction or the whole extract, with no cytotoxic effect against human lymphocytes or A. salina. The active compounds were identified as tannins, quinones, flavonoids and sesquiterpenlactones. In conclusion, active compounds against $T$. cruzi were identified for the first time in H. brasiletto, with non-cytotoxic effects. The $H$. brasiletto extract, according to our results, could be used as an alternative treatment for the Chagas disease; however, additional studies will be necessary to test their activity and doses in a murine model, with the complete identification of the active compounds, on which we are investigating. Rev. Biol. Trop. 65 (4): 1459-1469. Epub 2017 December 01.
\end{abstract}

Key words: Trypanosoma cruzi, Haematoxylum brasiletto, medicinal plants, methanolic extracts.

La tripanosomiasis americana es una enfermedad potencialmente mortal causada por el protozoario Trypanosoma cruzi (Chagas, 1909). Las zonas endémicas abarcan 21 países de América Latina, donde el parásito se transmite principalmente por vía vectorial (Calvo, 2015). La enfermedad presenta dos fases; el inicio de la infección es la fase aguda, y permanece hasta dos meses con parasitemia, fiebre y malestar. La etapa crónica, que se desarrolla después de algunos años en el 25-35\% de las personas infectadas, se caracteriza por no presentar parasitemia; en esta fase, los parásitos se alojan y reproducen en nidos de amastigotes de órganos y sistema nervioso periférico, de los cuales un $30 \%$ desarrolla desórdenes cardíacos y un $10 \%$, trastornos nerviosos y digestivos como el megacolon o megaesófago (Martino, 2012). Actualmente, los movimientos migratorios la han diseminado desde las regiones endémicas hasta Canadá y varios países europeos (Slot, Hogema, Molier, Bart, \& Zaaijer, 2016), 
en los cuales la WHO (2015) ha calculado entre seis y siete millones de personas infectadas.

Los dos únicos medicamentos tradicionales para tratar la enfermedad son el benznidazol y nifurtimox, ambos eficaces si se administran en la etapa aguda, pero su eficacia disminuye si se administra en la fase crónica. En la fase aguda de la enfermedad de Chagas se establecen cifras serológicas de curación cercanas al $100 \%$ (Fabbro et al., 2013), pero en la fase crónica se ha estimado que tan solo $5.9 \%$ de los pacientes que reciben tratamiento alcanzan a curarse (Matta, Gutierrez, Nascimiento, DoValle, \& Silva, 2012). Las reacciones adversas frecuentemente son causa de suspensión del tratamiento, pueden presentarse hasta en el $40 \%$ de los pacientes tratados con benznidazol y el $61 \%$ de los tratados con nifurtimox (Carrilero, Murcia, Martinez-Lage, \& Segovia, 2011; Murcia, Carrilero, Viñas, \& Segovia, 2012). El costo del medicamento es elevado para los gobiernos de los países en vías de desarrollo o con economía insuficiente en programas de salud, por ejemplo, en Colombia el costo anual se ha estimado en alrededor de 267 millones de dólares (WHO, 2015). Otra desventaja de los tratamientos tradicionales es la diferente susceptibilidad que han desarrollado los parásitos a estos compuestos, debido a la regulación de la expresión del gen de la adenina fosforibosiltransferasa (APRT), de la enzima lipoamido deshidrogenasa y de la tryparedoxina peroxidasa en cepas naturalmente resistentes (dos Santos et al., 2016; Kohatsu et al., 2016; García-Huertas, Mejía-Jaramillo, González, \& Triana-Chavez, 2017) por lo cual, la parasitemia no puede ser eliminada; además, la respuesta terapéutica varía en función del área geográfica, debido al linaje de las cepas de $T$. cruzi infectantes, dado que se ha descrito diferente susceptibilidad de algunas cepas de $T$. cruzi al tratamiento con benznidazol, por lo que la búsqueda de nuevos tratamientos tiene una alta prioridad (Villarreal, Barnabé, Sereno, \& Tibayrenc, 2004; Mejía-Jaramillo, Fernandez, Montilla, Nicholls, \& Triana-Chavez, 2012).

La fitoterapia representa la forma más antigua de los tratamientos terapéuticos en el ámbito mundial, en particular, las plantas son comúnmente usadas por la población mexicana para tratar infecciones bacterianas, micóticas y parasitarias. Por este motivo, nuestro grupo de investigadores reportó recientemente los resultados de extractos metanólicos de plantas de uso medicinal tradicional contra la cepa CL Brener de T. cruzi (Molina-Garza, BazaldúaRodríguez, Quintanilla-Licea, \& Galaviz-Silva, 2014), los cuales fueron obtenidos de Artemisia mexicana (syn. Artemisia ludoviciana var. mexicana Willd ex Spreng 1945) (Asteraceae) y Cymbopogon citratus (Stapf, 1906) (Andropogonodae), las cuales se usan contra el dolor de estómago, infecciones gastrointestinales y parásitos (Navarro, Villarreal, Rojas, \& Lozoya, 1996), Castela texana (Torr \& Gray, 1909) Rose (syn. C. tortuosa Liebm, 1853) (Simaroubaceae) en la disentería amebiana (Monzote, Alarcón, \& Setzer, 2012), Lippia graveolens (Kunth, 1818) (Verbenaceae) en los casos de desórdenes gastrointestinales y giardiasis (Monzote et al., 2012); Persea americana (Mill, 1768) (Lauracea), la cual se usa como tratamiento de infecciones micóticas (Wang et al., 2004) y como nematicida (Dang et al., 2010) Ruta chalepensis (Linnaeus, 1767) (Rutaceae), que se considera un buen antihelmíntico y espasmolítico (Günaydin \& Savci, 2005), Eryngium heterophyllum (Engelm, 1848) (Apiaceae), como tratamiento para diarrea, dolor de estómago fiebre y colelitiasis (Camou-Guerrero, Reyes-García, Martínez-Ramos, \& Casas, 2008), Haematoxylum brasiletto (Karst, 1862) (Fabaceae) para tratar infecciones bacterianas (Rosas-Piñón et al., 2012), Marrubium vulgare (Linnaeus, 1753) (Lamiaceae), usado como hipotensivo (Bardai, Lyoussi, Wibo, \& Morel, 2001) y Schinus molle (Linnaeus, 1753) (Anacardiaceae) que es un tratamiento antiespasmódico e hipotensivo (Yueqin et al., 2003). En el estudio previo, Molina-Garza et al. (2014), concluyen que los extractos de las últimas cuatro plantas exhibieron la mayor actividad e inhibieron el crecimiento entre el 88 y $100 \%$; incluso, cabe mencionar que se ha experimentado con un amplio espectro de productos naturales 
contra T. cruzi, pero muy pocos han sido útiles a una concentración inhibitoria recomendada del $50 \%\left(\mathrm{CI}_{50}\right)$ de $10 \mu \mathrm{g} / \mathrm{mL}$, en referencia a la $\mathrm{CI}_{50}$ para nifurtimox y para benidazol, la cual debe ser menor a los $3 \mu \mathrm{g} / \mathrm{mL}$ (Werner \& Zulantay, 2011), por lo cual fueron seleccionados para continuar con este proyecto.

El presente estudio, como continuación del anterior, tuvo como objetivos: 1- determinar la eficiencia de los extractos de las cuatro plantas seleccionadas contra una cepa geográficamente diferente de $T$. cruzi para el uso terapéutico en la región, aislado de vectores triatominos provenientes del estado de Nuevo León, México, debido a la alta variabilidad genética de las cepas de $T$. cruzi, y 2-identificar los compuestos activos que presenten la mayor inhibición del T. cruzi in vitro.

\section{MATERIALES Y MÉTODOS}

Material vegetal: Se recolectaron 15 ejemplares de $H$. brasiletto, C. citratus, $M$. vulgare y $S$. molle de tres diferentes viveros del área metropolitana del estado de Nuevo León, México (2540'17' N \& $100^{\circ} 18^{\prime} 0^{\prime \prime}$ W), a una altitud promedio de $500 \mathrm{msnm}$ de mayo a junio del 2014, a finales de primavera y parte del verano y con una temperatura anual que oscila entre los $20^{\circ} \mathrm{C}$ con precipitaciones de $650 \mathrm{~mm}$, con un clima que se considera semiárido cálido (INEGI, 2015). En este período, las plantas se encuentran en su mejor estado fisiológico y reproductivo, por ser temporada de lluvias y tener los fotoperiodos más largos del año. Los ejemplares frescos recolectados se colocaron en bolsas de plástico con hielo para transportarlos; la identificación se realizó en el Departamento de Botánica de la Facultad de Ciencias Biológicas de la UANL donde se depositaron los boucher con el registro de $H$. brasiletto (NL-0548), C. citratus (NL-5542), M. vulgare (NL-5555) y $S$. molle (NL-5567).

Preparación de los extractos: Se descartaron las plantas que tuvieran signos de infección o enfermedad, después se lavó con agua bidestilada estéril para eliminar las impurezas superficiales al chorro de agua; posteriormente, se procedió a secarlo a una temperatura de $30{ }^{\circ} \mathrm{C}$ por $24 \mathrm{~h}$ en una estufa (Cole Parmer, Inc.), una vez seco, se pulverizó con un molino Wiley, hasta obtener polvo fino. Se utilizaron diversas porciones de los ejemplares, como la parte aérea, tallos y hojas (Molina-Garza et al., 2014). Se pesaron $50 \mathrm{~g}$ del material vegetal molido y se depositó en un cartucho de celulosa (Cartucho Whatman 33 x $80 \mathrm{~mm}$ ) para la extracción con el aparato Soxhlet con $250 \mathrm{~mL}$ de metanol absoluto (J. T. Baker) como solvente de extracción, se colocó a reflujo a $60{ }^{\circ} \mathrm{C}$ por 40 h. Posteriormente, se filtró el extracto con papel Whatman $\mathrm{N}^{\circ} 1$ y se concentró con el rotavapor Buchi R-205. Se analizó el rendimiento de cada planta previo a realizar las pruebas contra el parásito (g de extracto/100 g de planta) (Quintanilla-Licea et al., 2012).

Parásitos: La actividad de los extractos fue evaluada en los epimastigotes de la cepa de T. cruzi obtenida a partir de las heces de Triatoma gerstaeckeri de la localidad de Cerralvo, Nuevo León (por la primera autora, KCPT), la cepa se cultivó en nuestro laboratorio en medio LIT (Liver infusion tryptose) con suero fetal bovino al $10 \%$, para incubarse a $28{ }^{\circ} \mathrm{C}$ y ser cosechada en la fase de crecimiento exponencial (Molina-Garza et al., 2014).

Evaluación de la actividad tripanosomicida de los extractos: Se realizó una solución de cada extracto metanólico de $15 \mathrm{mg}$ disueltos en dimetil sulfoxido $1 \%$ (DMSO) e incubados por $24 \mathrm{~h}$. Los bioensayos se realizaron por duplicado; para cada extracto se tomó como unidad experimental una microplaca de 96 pozos, donde se depositaron $200 \mu \mathrm{L}$ de la suspensión del medio LIT con $1.5 \times 10^{6}$ parásitos $/ \mathrm{mL}$, en cada pozo se agregó cada una de las cinco diferentes concentraciones del extracto con tres réplicas de cada uno de los tratamientos $(1000 \mu \mathrm{g} / \mathrm{mL}, 750 \mu \mathrm{g} / \mathrm{mL}$, $500 \mu \mathrm{g} / \mathrm{mL}, 250 \mu \mathrm{g} / \mathrm{mL}$ y $100 \mu \mathrm{g} / \mathrm{mL}$ ); simultáneamente, se colocaron el control positivo y negativo, se utilizó nifurtimox (Sigma-Aldrich) a $10 \mu \mathrm{g} / \mathrm{mL}$ y medio de cultivo con parásitos 
respectivamente, con un volumen igual al de los extractos de DMSO 1 \% (Muelas-Serrano, Nogal-Ruiz, \& Gómez-Barrio, 2000). Las concentraciones expresadas en $\mu \mathrm{g} / \mathrm{mL}$ de los extractos se prepararon en una balanza analítica con capacidad de $80 \mathrm{~g}$ a $0.0001 \mathrm{~g}$ (Nimbus $84 \mathrm{i}$, Adam Equipment) y mediante diluciones con micropipetas (Eppendorf) con capacidad de 1 $\mathrm{mL}$ a $0.5 \mu \mathrm{L}$. Las microplacas se incubaron (Memmert, Inc.) por 96 horas a $27{ }^{\circ} \mathrm{C}$ (Pizzolatti, Koga, Grisard, \& Steindel, 2002). El número de epimastigotes vivos se determinó mediante recuento en cámara de Neubauer. Los resultados se expresan como la concentración inhibitoria al $50 \%\left(\mathrm{CI}_{50}\right)$, que corresponde a la concentración a la cual ocurre el $50 \%$ de muerte celular. Se seleccionó el extracto con mayor actividad para identificar las familias funcionales mediante pruebas químicas coloridas (Pérez-Treviño, Molina-Garza, \& Galaviz-Silva, 2016). Se determinó la $\mathrm{CI}_{50}$ de los extractos contra la cepa de $T$ cruzi, mediante un análisis de regresión dosis-respuesta con el programa SPSS 17.0 (Al-Adhroey, Nor, Al-Mekhalfi, \& Mahumud., 2010) y con un intervalo de confianza (IC) del $95 \%$.

\section{Determinación de la actividad citotóxica} de los extractos en Artemia salina: El ensayo consistió en exponer grupos de larvas a las $48 \mathrm{~h}$ después de eclosionar, a las mismas concentraciones de extracto que se utilizaron contra $T$. cruzi $(1000 \mu \mathrm{g} / \mathrm{mL}, 750 \mu \mathrm{g} / \mathrm{mL}, 500 \mu \mathrm{g} / \mathrm{mL}$, $250 \mu \mathrm{g} / \mathrm{mL}$ y $100 \mu \mathrm{g} / \mathrm{mL})$ durante $24 \mathrm{~h}$ a temperatura ambiente y bajo régimen continuo de luz. Primero, se prepararon placas de 96 pozos, se añadió a cada uno $200 \mu \mathrm{L}$ de agua de mar suplementada con extracto de levadura y con diluciones del extracto correspondiente. Posteriormente, se transfirieron 10 larvas a cada pozo. Al finalizar las $24 \mathrm{~h}$ de exposición, se contabilizó el número de organismos muertos y se realizó una prueba PROBIT. Las larvas se consideraron muertas si no exhibían movimiento durante varios segundos de observación al microscopio estereoscópico. El experimento se consideró válido si el porcentaje de mortalidad en los controles no excedió de $10 \%$ (Fernández-Calienes Valdez et al., 2009).

Fraccionamiento del extracto: Para separar los compuestos presentes en los extractos, se realizó cromatografía de capa fina, se utilizaron placas de sílica gel en soporte (TLC Sílica gel 60 Sigma Aldrich). La observación de la separación de los compuestos se realizó bajo la luz visible y ultravioleta (365 nm), en este último caso con el empleo de una lámpara portátil. Se realizó una separación mediante una fase móvil $(\mathrm{v} / \mathrm{v})$ de cloroformo: metanol (8:2) absolutos, tras evaluar las fracciones, se conservaron las dos con actividad tripanosomicida mayor, identificadas mediante su factor de retención ( $\mathrm{Rf})$, obtenido a partir de la fórmula $\mathrm{R}_{\mathrm{f}}=$ Distancia del compuesto desde el origen/ distancia del solvente desde el origen (TomásAlonso, 1993). Las fracciones obtenidas se denominaron Fr21 (Rf: 0.25) y Fr22 (Rf: 0.8), mismas que fueron probadas contra T. cruzi, al siguir la metodología antes descrita (Murillo, Castro, Chavarría, \& Poveda, 2006; Volonté \& Quiroga, 2013; Mijares-Bullaín, Torres-Rodriguez, \& Hermosilla-Espinosa, 2014).

Ensayo de citotoxicidad de la fracción
con mayor actividad tripanosomicida en
linfocitos humanos: Se emplearon linfocitos humanos de sangre periférica de un donante joven (26 años) no fumador, sin haber ingerido alcohol un mes antes de la donación y quien firmó el consentimiento informado para participar en este estudio. Los linfocitos se aislaron por el método tradicional en gradiente (Boyum, 1984). Se recolectaron $5 \mathrm{~mL}$ de sangre en un tubo Vacutainer con heparina, se mezcló por inversión con $5 \mathrm{~mL}$ de amortiguador de fosfato de sodio (PBS) en proporción 1:1, se transfirió lentamente por las paredes, evitando la formación de turbulencia, en $10 \mathrm{~mL}$ de Ficoll@Paque Plus (Sigma-Aldrich), se verificó la formación de dos capas y se centrifugó durante $30 \mathrm{~min}$ a $3000 \mathrm{rpm}$ a $4^{\circ} \mathrm{C}$. Se extrajo con precaución la capa de linfocitos y se transfirió a otro tubo limpio y seco. Finalmente, se centrifugó para descartar el PBS y se agregó $1 \mathrm{~mL}$ de medio 
modificado Dulbecco de Eagle (DMEM, Sigma-Aldrich). Se determinó la viabilidad inicial mediante la técnica de exclusión por azul de tripano, se incubaron en el mismo medio a $37^{\circ} \mathrm{C}$ por $2 \mathrm{~h}$. Posteriormente, se evaluaron las concentraciones de $0.15,0.30,0.45$ y $0.6 \mathrm{mg} /$ $\mathrm{mL}$ de la fracción con mayor actividad tripanosomicida. Como control positivo se utilizó concavalina A. La prueba se realizó en una solución de $1 \times 10^{6}$ linfocitos $/ \mathrm{mL}$, se cultivaron en medio DMEM y se colocaron en una microplaca con un volumen final de $200 \mu \mathrm{L}$. Se incubaron a $37^{\circ} \mathrm{C}$ por $4 \mathrm{~h}$. Posteriormente, se evaluó la viabilidad celular mediante la formación de cristales de formazán, por medio del método de la reducción metabólica del Bromuro de 3-(4,5-dimetiltiazol-2-ilo)-2,5-difeniltetrazol (MTT) (Cho et al., 2016).

Determinación cualitativa de metabolitos secundarios: Se realizó la identificación de metabolitos secundarios mediante reacciones químicas coloridas. La determinación de flavonoides se efectuó mediante la prueba de Shinoda; las cumarinas mediante hidróxido de sodio $10 \%$; se utilizó la prueba de Dragendorff para determinar la presencia de alcaloides; los esteroles y terpenos se identificaron mediante el ensayo de Lieberman-Buchard; la prueba de Balje se utilizó para analizar la presencia de sesquiterpenlactonas; quinonas y taninos mediante ácido sulfúrico y cloruro férrico $1 \%$, respectivamente; la determinación de saponinas se realizó mediante la prueba de la espuma (Ortega, Benitez-Bampo, \& Cabezas-Fajardo, 2011; Rathore, Bhatt, Dhyani, \& Jain, 2012; Sotomayor, 2014).

\section{RESULTADOS}

El extracto completo $H$. brasiletto fue el que tuvo mayor rendimiento con $21.3 \%$; en orden decreciente se encontró a $S$. molle (17.8\%), M. vulgare (16.3\%), y finalmente, C. citratus $(14.8 \%)$.

El extracto de $H$. brasiletto presentó la mayor actividad inhibitoria contra $T$. cruzi a una concentración de $1 \mathrm{mg} / \mathrm{mL}$ (90\%) con una $\mathrm{CI}_{50}$ de $0.543 \mathrm{mg} / \mathrm{mL}$ : Este fue seguido por el extracto de $M$. vulgare, el cual también presentó buena actividad tripansomicida $\left(\mathrm{CI}_{50}=\right.$ 0.647 ) con baja toxicidad en A. salina. Aunque el extracto de $C$. citratus fue el menos tóxico en el modelo de Artemia, éste no presentó una buena actividad tripanosomicida; los extractos restantes tampoco mostraron una toxicidad notable anti $T$. cruzi (Cuadro 1). Con los controles positivos, la $\mathrm{CI}_{50}$ del nifurtimox $(10 \mu \mathrm{g} / \mathrm{mL})$ presentó una inhibición del $80 \%$ de los parásitos. En los controles negativos no se encontró efecto sobre los epimastigotes de $T$. cruzi.

CUADRO 1

Evaluación in vitro de la actividad tripanosomicida de los extractos metanólicos de plantas y su toxicidad en modelo de Artemia salina

TABLE 1

In vitro assessment of the trypanosomicidal activity of metanolic extracts from four plants and toxicity assay in a model of Artemia salina

\begin{tabular}{lcccc}
\multicolumn{1}{c}{ Especie de planta } & Parte estudiada & \multicolumn{2}{c}{ Trypanosoma cruzi } & Artemia salina \\
& & \% inhibición & $\mathrm{CI}_{50}$ & $\mathrm{CI}_{50}$ \\
Haematoxylum brasiletto & Corteza & 90 & $0.543(0.446-0.635)$ & $2.412(1.731-4.659)$ \\
Cymbopogon citratus & Aérea & 2 & $1.21(1.05-1.40)$ & $3.148(2.129-6.300)$ \\
Marrubium vulgare & Aérea & 84 & $0.647(0.521-0.776)$ & $2.115(1.554-3.974)$ \\
Schinus molle & Hojas & 68 & $0.827(0.712-0.957)$ & $2.279(1.648-4.368)$ \\
Nifurtimox (Control) & NA & 80 & NA & NA \\
\hline
\end{tabular}

$\%$ de inhibición= porcentaje de inhibición a $1 \mathrm{mg} / \mathrm{mL} . \mathrm{CI}_{50}=$ Concentración inhibitoria $(\mathrm{mg} / \mathrm{mL})$ que causa la muerte celular del $50 \%$ de los protozoarios a intervalo de confianza del $95 \%$. NA= No Aplica. 
La fracción Fr22 de H. brasiletto presentó una $\mathrm{CI}_{50}$ de $0.238 \mathrm{mg} / \mathrm{mL}(\mathrm{IC}=0.174-0.301)$, la cual fue incluso más eficiente que el extracto completo (Cuadro 2). Por lo cual esta fracción fue la única seleccionada para determinar el ensayo de citotoxicidad en linfocitos humanos para determinar la selectividad de la misma. Esta fracción Fr22, a dosis de $0.6 \mathrm{mg} / \mathrm{mL}$ (la más concentrada) presentó un porcentaje de

\section{CUADRO 2}

Desafíos de las fracciones de Hematoxylum brasiletto contra T. cruzi

TABLE 2

Challenge of the fractions from Hematoxylum brasiletto against T.cruzi

\begin{tabular}{lccc} 
Región $^{1}$ & $\begin{array}{c}\text { Concentración } \\
\text { inhibitoria }^{2}\end{array}$ & $\begin{array}{c}\text { Dosis en } \\
\mathrm{mg} / \mathrm{mL}\end{array}$ & IC al 95 \% \\
Fr21 & $\mathrm{CI}_{80}$ & 0.627 & $0.530-0.784$ \\
& $\mathrm{CI}_{50}$ & 0.372 & $0.306-0.454$ \\
Fr22 & $\mathrm{CI}_{80}$ & 0.493 & $0.415-0.614$ \\
& $\mathrm{CI}_{50}$ & 0.238 & $0.174-0.301$ \\
\hline
\end{tabular}

${ }^{1}$ Fr21 = Fracción a tiempo de retención de 0.25, Fr22 = Fracción a tiempo de retención de 0.8 .

${ }^{2} \mathrm{CI}_{50}=$ Concentración mínima para inhibir el $50 \%$ de los parásitos.

$\mathrm{CI}_{80}=$ Concentración mínima para inhibir el $80 \%$ de los parásitos.

IC =Intervalo de confianza viabilidad de linfocitos del $81 \%$. Mientras que el efecto inhibidor medio de la fracción Fr22 contra los epimastigotes, se presentó a los 0.24 $\mathrm{mg} / \mathrm{mL}$, la cual fue marcadamente menor respecto al efecto citotóxico.

Posterior a la separación, se procedió a identificar las familias de compuestos presentes en cada fracción, así como en el extracto completo, lo cual permitió identificar como compuestos activos a los flavonoides, sesquiterpenlactonas y quinonas (Cuadro 3).

\section{DISCUSIÓN}

Los extractos de plantas con antecedentes de uso medicinal han sido un referente constante en el tratamiento de la enfermedad de Chagas debido a los efectos adversos del benznidazol y el nifurtimox (Cuhna et al., 2003; Paveto et al., 2004; Abdel-Sattar, Maes, \& Salama, 2010). Desde que se observó que existe una correlación positiva entre la actividad tripanosomicida contra epimastigotes in vitro y la actividad contra tripomastigotes in vivo, el uso de epimastigotes para estimar la actividad tripanosomicida se ha difundido más rápidamente (Schlemper, Chiari \& Brener, 1977; Abe et al., 2002).

El extracto de $S$. molle ha sido utilizado ampliamente como repelente de insectos,

CUADRO 3

Compuestos identificados en extractos de Haematoxylum brasiletto mediante pruebas coloridas

TABLE 3

Identification of compounds from extracts of Haematoxylum brasiletto by colored chemical reactions

\begin{tabular}{llccc}
\multicolumn{1}{c}{ Familia de compuestos } & \multicolumn{1}{c}{ Prueba } & Completo $^{1}$ & Fr2 $^{1}$ & Fr22 \\
Taninos & Cloruro férrico 1\% & + & - & + \\
Flavonoides & Prueba de Shinoa & + & + & + \\
Esteroles y triptenos & Lieberman-Burchard & + & - & - \\
Cumarinas & Hidróxido de Sodio $10 \%$ & - & - & - \\
Sesquiterpenlactonas & Prueba de Balje & + & - & + \\
Alcaloides & Dragendorff & - & - & - \\
Saponinas & Espuma & - & - & - \\
Quinonas & Ácido sulfúrico & + & - & + \\
\hline
\end{tabular}

Fr2 $=$ Fracción a tiempo de retención de 0.25 .

Fr22 = Fracción a tiempo de retención de 0.8

$1_{+}=$presencia del metabolito, $-=$ausencia del metabolito. 
incluyendo para Triatoma infestans, vector de la enfermedad de Chagas, identificándose hasta 65 compuestos con actividad repelente, como son el limoneno, eucaliptol y terpenos; sin embargo, su uso antiparasitario se ha restringido solo al extracto completo (Ferrero, González, \& Chopa, 2006; Abdel-Sattar et al., 2010; Molina-Garza et al., 2014). Del extracto de $M$. vulgare se han identificado cinco compuestos de una fracción polar con actividad anti-inflamatoria no esteroidea: el ácido cafeoil-L-málico, verbascosido, forsitosida, arenariosido y ballotetrosido; de los cuales sólo los dos primeros compuestos se han reportado con actividad antibiótica importante (Sahpaz, Garbacki, Tits, \& Bailleul, 2002). Otros 29 compuestos identificados con actividad biológica en el aceite escencial de C. citratus incluyen principalmente al mirceno, citronelal, R-limoneno, citral y $\beta$-citronelol, que presentan una buena actividad individual contra T. brucei brucei (Kpoviessi et al., 2014). Sin embargo, no han sido probados contra T. cruzi, lo cual abre nuevas expectativas para futuros estudios y determinar si el efecto será igual de efectivo.

De los extractos analizados, las dosis (mg/ $\mathrm{mL}$ ) en las que se observaron las óptimas actividades anti-T. cruzi $\left(\mathrm{CI}_{50}\right)$ fueron 1.21 para $C$. citratus; 0.647 para $M$. vulgare y 0.827 en el caso de $S$. molle, pero fue $H$. brasiletto (conocido como palo de Brasil en esta región), el que presentó una actividad marcada a los $543 \mu \mathrm{g} /$ $\mathrm{mL}$. Otros resultados parciales se presentaron en el LI Congreso Nacional de la Sociedad Mexicana de Entomología, pero este estudio muestra los resultados completos (Pérez-Treviño et al., 2016).

En reporte anteriores, se describen pruebas preliminares con 20 familias y 37 especies de plantas de uso medicinal de México y Guatemala contra epimastigotes de $T$. cruzi, reportándose la "fijación" del parásito a dosis de 2 $\mathrm{mg} / \mathrm{mL}$ con la inmovilización del mismo en un $90-80 \%$ a dosis de $1 \mathrm{mg} / \mathrm{mL}$ con el extracto de $H$. brasiletto, sin embargo, no se mencionan los compuestos activos (Abe et al., 2002). Así mismo, el efecto antibiótico de $H$. brasiletto ha sido probado contra bacterias como Escherichia coli y Staphylococcus aureus; pero con mejores resultados contra el último (Yasunaka et al., 2005). El extracto de $H$. brasiletto también ha sido probado contra 12 cepas de bacterias antibiótico resistentes y un eucariota (Candida albicans); y fueron identificados ocho compuestos con actividad biológica: hematoxilina, brazileina, floroglucinol, 5-metoxipsoraleno, metil galato, ácido gálico, ácido 4-hidroxicinamico y ácido caféico (Rivero-Cruz, 2008).

En el extracto de palo de Brasil se encontró la presencia de flavonoides, compuestos que en estudios previos han sido asociados con actividad contra diversos géneros de parásitos cómo Plasmodium, Leishmania, Entamoeba, Giardia, Trichomonas y Trypanosoma; además de características antioxidante (MartínezFlórez, González-Gallego, \& Culebras, 2002). También se han identificado quinonas y triterpenos reportados con actividad antimicrobiana (Muñoz-Jáuregui, Ramos-Escudero, AlvaradoOrtiz, \& Castañeda-Castañeda, 2007; Ramírez, Mendoza, Arreola, \& Ordaz, 2010), así como también en el presente estudio, identificamos la presencia de sesquiterpenlactonas, las cuales se han reportado con actividad contra los parásitos de la malaria y la leishmaniasis en ensayos in vitro (Oketch-Rabah et al., 1998; Sülsen, 2012; Cano de Terrones, 2014; Galarraga, Luis, Rojas, Offer, \& Dobois, 2014).

Con respecto al modelo de citotoxicidad con A. salina descrito en este estudio, este ha sido ampliamente utilizado en toxicología para evaluar los riesgos del uso de diversas sustancias, entre ellas los extractos de plantas, por ser una técnica fácil y económica, que además puede orientar sobre la toxicidad de muchos compuestos (Pino-Pérez \& Jorge-Lazo, 2010). Sin embargo, al comparar datos de toxicidad obtenidos de A. salina frente a cultivo celular, se aprecia que la $\mathrm{CI}_{50}$ resulta mayor, lo cual posiblemente sea debido a la mayor complejidad bioquímica de las larvas, por lo que se hace necesario el uso de células para evaluar la toxicidad de un compuesto (Vega-Menchaca et al., 2013; Espitia-Baena, RobledoRestrepo, Cuadrado-Cano, Duran-Sandoval, \& Gómez-Estrada, 2014). Los compuestos 
presentes en el extracto de $H$. brasiletto abren una nueva alternativa como una posible vía de tratamiento, de acuerdo a los resultados obtenidos con la fracción Fr22. Es importante verificar mediante ensayos de toxicidad con $A$. salina y linfocitos humanos, si su uso se puede dirigir a organismos superiores, similar a los resultados aquí descritos y los reportados por Déciga-Campos et al. (2007), H. brasilleto es una planta no tóxica.

En conclusión, este trabajo presenta la primera caracterización parcial del extracto de $H$. brasiletto y la evaluación de la capacidad tripanosomicida de cada fracción del extracto, además de ser la primera evaluación de toxicidad en un modelo más complejo, cómo lo es en linfocito humano, por lo que se recomienda estudios más detallados para identificar la(s) molécula(s) responsable(s) de la actividad. De acuerdo con nuestros resultados, el extracto de $H$. brasiletto podría ser usado como tratamiento alternativo para la enfermedad de Chagas, sin embargo, se necesitan estudios adicionales para probar su actividad y dosis en un modelo murino con la completa identificación de los compuestos activos, sobre los cual se está investigando. También se concluye la presencia y la actividad tripanosomicida de la familia de las sesquiterpenlactonas; y se consolida la capacidad tripanosomicida del extracto de $M$. vulgare para futuros estudios de nuevas estrategias para el tratamiento de la enfermedad de Chagas.

\section{AGRADECIMIENTOS}

Este estudio se efectuó con financiamiento del proyecto PAICyT-UANL (CT305-15) "Evaluación de la actividad antiparasitaria de extractos metanólicos de plantas con uso medicinal sobre la cepa regional de Trypanosoma cruzi" (ZJMG), y al proyecto "Zoonosis Parasitarias" Clave CT-305-15. UANL-CA-278 de PROMEP-SEP N ${ }^{\circ}$ 103.5/11/1047 P/CA (LGS).

\section{RESUMEN}

La tripanosomiasis americana es una enfermedad potencialmente letal causada por el protozoario hemoflagelado Trypanosoma cruzi. Esta enfermedad desatendida afecta entre 6 a 7 millones de personas en el mundo. Actualmente existen solo dos medicamentos para tratar la enfermedad, benznidazol y nifurtimox, ambos efectivos si se administran en la fase aguda, aunque su actividad disminuye en la fase crónica, también induce efectos secundarios significativos. El objetivo de este estudio fue analizar la actividad tripanosomicida de los extractos metanólicos de Hematoxilum brasiletto, Marrubium vulgare, Schinus molle, and Cympongon citratus contra T. cruzi epimastigotes, seguido de la separación cromatográfica e identificación de los compuestos activos del mejor candidato por medio de reacciones químicas coloridas y también, determinar su efecto citotóxico en cultivos de linfocitos humanos y Artemia salina. El extracto de H. brasiletto presentó la mejor actividad anti- $T$. cruzi con una concentración inhibitoria $(\mathrm{CI})$ de $543 \mu \mathrm{g} / \mathrm{mL}$; seguida en orden descendiente por $M$. vulgare $\left(\mathrm{CI}_{50}=647 \mu \mathrm{g} / \mathrm{mL}\right)$, S. molle $(\mathrm{CI}=827 \mu \mathrm{g} / \mathrm{mL})$ y finalmente, C. citratus $(\mathrm{CI}=1210 \mu \mathrm{g}$ ) $\mathrm{mL})$. La fracción cromatográfica Fr22 de H. brasiletto presentó la mejor efectividad tripanosomicida $(\mathrm{CI}=0.238 \mathrm{mg} /$ $\mathrm{mL}$ ) que las otras fracciones o el extracto completo, además no presentaron efecto citotóxico contra linfocitos o $A$. salina. Los compuestos activos fueron identificados como taninos, quinonas, flavonoides y sesquiterpenlactonas. En conclusión, fueron identificados los compuestos activos contra T. cruzi en H. brasiletto los cuales no tuvieron efecto citotóxico. De acuerdo a nuestros resultados, el extracto de $H$. brasiletto podría ser usado como tratamiento alternativo para la enfermedad de Chagas, sin embargo, se necesitan estudios adicionales para probar su actividad y dosis en un modelo murino con la completa identificación de los compuestos activos, sobre los cual se está investigando.

Palabras clave: Trypanosoma cruzi, Haematoxylum brasiletto, plantas medicinales, extractos metanólicos.

\section{REFERENCIAS}

Abdel-Sattar, E., Maes, L., \& Salama, M. M. (2010). In vitro activities of plant extracts from Saudi Arabia against malaria, leishmaniasis, sleeping sickness and Chagas disease. Phytotherapy Research, 24, 1322-1328.

Abe, F., Nagafuji, S., Yamauchi, T., Okabe, H., Maki, J., Higo, H., Akahane, H., Aguilar, A., Jiménez-Estrada, M., \& Reyes-Chilpa, R. (2002). Trypanocidal constituents in plants. 1. Evaluation of some Mexican plants 
for their trypanocidal activity and active constituents in Guaco, roots of Aristolochia taliscana. Biological and Pharmaceutical Bulletin, 25, 1188-1191.

Al-Adhroey, A. H., Nor, Z. M., Al-Mekhlafi, H. M., \& Mahmud, R. (2010). Median lethal dose, antimalarial activity, phytochemical screening and radical scavenging of methanolic Lenguas galanga rhizome extract. Molecules, 15, 8366-8376.

Bardai, S. E., Lyoussi, B., Wibo, M., \& Morel, N. (2001). Pharmacological evidence ofhypotensive activity of Marrubium vulgare and Foeniculum vulgare in sponta-neously hypertensive rat. Clinical and Experimental Hypertension, 23, 329-343.

Boyum, A. (1984). Separation of lymphocytes, granulocytes, and monocytes from human blood using iodinated density gradient media. Methods in Enzymology, 108, 88-102.

Calvo, M. S. (2015). Tripanosomiasis americana o enfermedad de Chagas. Revista Médica de Costa Rica y Centroamerica, 71, 539-544.

Camou-Guerrero, A., Reyes-García, V., Martínez-Ramos, M., \& Casas, A. (2008). Knowledge and use value of plant species in a Rarámuri community: a gender perspective for conservation. Human Ecology, $36,259-272$.

Cano de Terrones, T. (2014). Caracterización de una espirolactona sesquiterpénica $\alpha$-metilénica obtenida de Ambrosia arborescens Miller y evaluación de su actividad biológica en Trypanosoma cruzi. Revista de la Sociedad Química del Perú, 80, 124-135.

Carrilero, B., Murcia, L., Martinez-Lage, L., \& Segovia, M. (2011). Side effects of benznidazole treatment in a cohort of patients with Chagas disease in nonendemic country. Revista Española de Quimioterapia, 24, 123-26.

Chagas, C. (1909). Nova tripanozomiaze humana: estudos sobre a morfolojia e o ciclo evolutivo do Schizotrypanum cruzi n. gen., n. sp., ajente etiolojico de nova entidade morbida do homem. Memórias do Instituto Oswaldo Cruz, 1, 159-218.

Cho, Y. H., Lee, J. W., Woo, H. D., Lee, S., Kim, Y. J., Lee, Y., Shin, S., Joung, H., \& Chung, H. W. (2016). Protective effect of onion extract on bleomycin-induced cytotoxicity and genotoxicity in human lymphocytes. International Journal of Environmental Research and Public Health, 13, 227.

Cunha, W. R., Martins, C., Ferreira, D. D., Crotti, A. E., Lopes, N. P., \& Albuquerque, S. (2003). In vitro trypanocidal activity of triterpenes from Miconia species. Planta Medica, 69, 470-471.

Dang, Q. L., Kwon, H. R., Choi, Y. H., Choi, G. J., Jang, K. S., Park, M. S., Lim, C. H., Ngoc, L. H., \& Kim, J. C. (2010). Nematicidal activity against Bursaphelenchus xylophilus ofisoobtusilactone a isolated from Persea americana. Nematology, 12, 247-253.

Déciga-Campos, M., Rivero-Cruz, I., Arriaga-Alba, M., Castañeda-Corral, G., Angeles-López, G. E., Navarrete, A., \& Mata, R. (2007). Acute toxicity and mutagenic activity of Mexican plants used in traditional medicine. Journal of Ethnopharmacology, $110,334-342$.

dos Santos, P. F., Moreira, D. S., Baba, E. H., Volpe, C. M., Ruiz, J. C., Romanha, A. J., \& Murta, S. M. (2016). Molecular characterization of lipoamide dehydrogenase gene in Trypanosoma cruzi populations susceptible and resistant to benznidazole. Experimental Parasitology, 170, 1-9. http://dx.doi.org/10.1016/j. exppara.2016.08.006

Espitia-Baena, J. E., Robledo-Restrepo, S. M., CuadradoCano, B., Duran-Sandoval, H. D. R., \& GómezEstrada, H. A. (2014). Perfil fitoquímico, actividad anti-Leishmania, hemolítica y toxicológica de Cordia dentata Poir y Heliotropium indicum L. Revista Cubana de Plantas Medicinales, 19, 208-224.

Fabbro, D., Velazquez, E., Bizai, M. L., Denner, S., Olivera, V., Arias, E., Pravia, C., \& Ruiz, A. M. (2013). Evaluation of the ELISA-F29 test as an early marker of therapeutic efficacy in adults with chronic Chagas disease. Revista do Instituto de Medicina Tropical de São Paulo, 55, 167-172.

Fernández-Calienes, A., Mendiola, J., Monzote, L., García, M., Sariego, I., Acuña, D., Ramón, L., \& Gutiérrez, Y. (2009). Evaluación de la toxicidad de extractos de plantas cubanas con posible acción antiparasitaria utilizando larvas de Artemia salina L. Revista Cubana de Medicina Tropical, 61, 254-258.

Ferrero, A. A., González, J. W., \& Chopa, C. S. (2006). Biological activity of Schinus molle on Triatoma infestans. Fitoterapia, 77, 381-383.

Galarraga, E., Luis, J. M. A., Rojas, L., Offer, A. C. M., \& Dubois, M. A. L. (2014). Triterpenos y saponinas triterpénicas de Phytolacca icosandra y Phytolacca rugosa. Ciencia, 22, 53-66.

García-Huertas, P., Mejía-Jaramillo, A. M., González, L., \& Triana-Chávez, O. (2017). Transcriptome and functional genomics reveal the participation of adenine fhosphoribosyl transferase in Trypanosoma cruzi resistance to benznidazole. Journal of Cellular Biochemistry, DOI 10.1002/jcb.25978 http://onlinelibrary.wiley.com/doi/10.1002/jcb.25978/full

Günaydin, K., \& Savci, S. (2005). Phytochemical studies on Ruta chalepensis (Lam.) Lamark. Natural Products Research, 19, 203-210.

Instituto Nacional de Estadística y Geografía (INEGI). (2015). Conociendo Nuevo León. Quinta edición. Recuperado de http://internet.contenidos.inegi.org. $\mathrm{mx} /$ contenidos/productos//prod_serv/contenidos/ 
espanol/bvinegi/productos/estudios/conociendo/702825213114.pdf

Kohatsu, A. A., Silva, F. A., Francisco, A. I., Rimoldi, A., Silva, M. T., Vargas, M. D., \& Cicarelli, R. M. (2016). Differential expression on mitochondrial tryparedoxin peroxidase (mTcTXNPx) in Trypanosoma cruzi after ferrocenyl diamine hydrochlorides treatments. The Brazilian Journal of Infectious Diseases. http:// dx.doi.org/10.1016/j.bjid.2016.10.010

Kpoviessi, S., Bero, J., Agbani, P., Gbaguidi, F., Kpadonou-Kpoviessi, B., Sinsin, B., Accrombessi, G., Frédérich, M., Moudachirou, M., \& Quetin-Leclercq, J. (2014). Chemical composition, cytotoxicity and in vitro antitrypanosomal and antiplasmodial activity of the essential oils of four Cymbopogon species from Benin. Journal of Ethnopharmacology, 151, 652-659.

Matta, P. M., Gutierrez, F. R., Nascimento, M. S., DoValle, M. A., \& Silva, J. S. (2012). Antiparasitical chemotherapy in Chagas' disease cardiomyopathy: current evidence. Tropical Medicine and International Health, 17, 1057-65.

Martínez-Flórez, S., González- Gallego, J., \& Culebras, J. M. (2002). Los flavonoides: propiedades y acciones antioxidantes. Nutrición Hospitalaria, 17, 271-278.

Martino, V. S. 2012. Problemática sanitaria y social de la enfermedad de Chagas. Aporte de la medicina tradicional argentina. Dominguezia, 28, 29-37.

Mijares-Bullaín, M., Torres-Rodriguez, E., \& HermosillaEspinosa, R. (2014). Tamizaje fitoquímico de los extractos de Faramea occidentalis (L.) A. Rich. (nabaco). Revista Cubana de Plantas Medicinales, 19, 421-432.

Mejía-Jaramillo, A. M., Fernández, G. J., Montilla, M., Nicholls, R. S., \& Triana-Chávez, O. (2012). Sensibilidad al benzonidazol de cepas de Trypanosoma cruzi sugiere la circulación de cepas naturalmente resistentes en Colombia. Biomédica, 32, 196-205.

Molina-Garza, Z. J., Bazaldúa-Rodríguez, A. F., Quintanilla-Licea, R., \& Galaviz-Silva, L. (2014). AntiTrypanosoma cruzi activity of 10 medicinal plants used in northeast Mexico. Acta Tropica, 136, 14-18. http://dx.doi.org/10.1016/j.actatropica.2014.04.006

Monzote, L., Alarcón, O., \& Setzer, W. N. (2012). Antiprotozoal activity of essential oils review. Agriculturae Conspectus Scientificus, 77, 167-175.

Muelas-Serrano, S., Nogal-Ruiz, J., \& Gómez-Barrio, A. 2000. Setting of a colorimetric method to determine the viability of Trypanosoma cruzi epimastigotes. Parasitology Research 86, 999-1002.

Muñoz-Jáuregui, A. M., Ramos-Escudero, D. F., AlvaradoOrtiz Ureta, C., \& Castañeda-Castañeda, B. (2007). Evaluación de la capacidad antioxidante y contenido de compuestos fenólicos en recursos vegetales promisorios. Revista de la Sociedad Química del Perú, 73, 142-149.

Murcia, L., Carrilero, B., Viñas, P. A., \& Segovia, M. (2012) Nifurtimox chemotherapy: collateral effects in treated Trypanosoma cruzi infected patients. Revista Española de Quimioterapia, 25, 1-3.

Murillo, R., Castro, V., Chavarría, M., \& Poveda, L. (2006). Lignanos de Zanthoxylum acuminatum. Revista de Ciencia y Tecnología, 24, 227-232.

Navarro, V., Villarreal, M. L., Rojas, G., \& Lozoya, X. (1996). Antimicrobial evaluation of some plants used in Mexican traditional medicine for the treatment of infectious diseases. Journal of Ethnopharmacology, $53,143-147$.

Oketch-Rabah, H. A., Christensen, S. B., Frydenvang, K., Dossaji, S. F., Theander, T. G., Cornett, C., Watkins, W. M., Kharazmi, A., \& Lemmich, E. (1998). Antiprotozoal properties of 16, 17-dihydrobrachycalyxolide from Vernonia brachycalyx. Planta Medica, 64, 559-562.

Ortega, N. S., Benitez-Bampo, N., \& Cabezas-Fajardo, F. A. (2011). Antibacterial activity and cualitative composition propolis from two climatic regions Cauca department. Biotecnología en el Sector Agropecuario y Agroindustrial, 9, 8-16.

Paveto, C., Güida, M. C., Esteva, M. I., Martino, V., Coussio, J., Flawiá, M. M., \& Torres, H. N. (2004). Anti-Trypanosoma cruzi activity of green tea (Camellia sinensis) catechins. Antimicrobial Agents and Chemotherapy, 48, 69-74.

Pérez-Treviño, K. C., Molina-Garza, Z. J., \& GalavizSilva, L. (2016) Evaluación de la actividad antitrypanosomal de extractos metanólicos de plantas con uso medicinal. Entomología Mexicana, 3, 656-659.

Pino-Pérez, O., \& Jorge-Lazo, F. (2010). Ensayo de Artemia: útil herramienta de trabajo para ecotoxicólogos y químicos de productos naturales. Revista de Protección Vegetal, 25, 34-43.

Pizzolatti, M. G., Koga, A. H., Grisard, E. C., \& Steindel, M. (2002). Trypanocidal activity of extracts from Brazilian Atlantic rain forest plant species. Phytomedicine, 10, 422-426.

Quintanilla-Licea, R., Morado-Castillo, R., Gomez-Flores, R., Laatsch, H., Verde-Star, M. J., HernándezMartínez, H., Tamez-Guerra, P., Tamez-Guerra, R., \& Rodríguez-Padilla, C. (2012). Bioassay-guided isolation and identification of cytotoxic compounds from Gymnosperma glutinosum leaves. Molecules, 17, 11229-11241.

Ramírez, M. E., Mendoza, J. A., Arreola, R. H., \& Ordaz, C. (2010). Flavonoides con actividad antiprotozoaria. Revista Mexicana de Ciencias Farmacéuticas, 41, 6-21. 
Rathore, S. K., Bhatt, S., Dhyani, S., \& Jain, A. (2012). Preliminary phytochemical screening of medicinal plant Ziziphus mauritiana Lam. fruits. International Journal of Current Pharmaceutical Review and Research, 4, 160-162.

Rivero-Cruz, J. F. (2008). Antimicrobial compounds isolated from Haematoxylon brasiletto. Journal of Ethnopharmacology, 119, 99-103.

Rosas-Piñón, Y., Mejía, A., Díaz-Ruiz, G., Aguilar, M S., Sánchez-Nieto, S., \& Rivero-Cruz, J. F. (2012). Ethnobotanical survey and antibacterial activity of plants usedin the Altiplane region of Mexico for the treatment of oral cavity infections. Journal of Ethnopharmacology, 141, 860-865.

Sahpaz, S., Garbacki, N., Tits, M., \& Bailleul, F. (2002). Isolation and pharmacological activity of phenylpropanoid esters from Marrubium vulgare. Journal of Ethnopharmacology, 79, 389-392.

Schlemper, B. R., Chiari, E., \& Brener, Z. 1977. Growthinhibition drug test with Trypanosoma cruzi culture forms. Journal of Protozology, 4, 544-547.

Slot, E., Hogema, B. M., Molier, M., Bart, A., \& Zaaijer, H. L. (2016). Risk Factors and Screening for Trypanosoma cruzi Infection of Dutch Blood Donors. PloS one, 11(3), 1-9. https://doi.org/10.1371/journal. pone. 0151038

Sotomayor, R. (2014). Extracción y cuantificación de antocianinas a partir de los granos de Zea mays L. (maíz morado). Ciencia y Desarrollo, 16, 69-74.

Sülsen, V. P. (2012). Búsqueda de compuestos antiprotozoarios en especies de la flora medicinal argentina. Dominguezia, 28, 19-27.

Tomás-Alonso, F. (1993). Química orgánica estructural. Vol. 5 de Manuales y materiales didácticos. Murcia, España: EDITUM.

Vega-Menchaca, M. D. C., Verde-Star, J., Oranday-Cárdenas, A., Morales-Rubio, M. E., Núñez-González, M.
A., Rivera-Guillén, M. A., Serrano-Gallardo, L. J., \& Rivas-Morales, C. (2013). Actividad antibacteriana y citotóxica de Leucophyllum frutescens (Berl) IM Johnst del Norte de México contra Staphylococcus aureus de aislados clínicos. Revista Mexicana de Ciencias Farmacéuticas, 44, 24-30.

Villarreal, D., Barnabé, C., Sereno, D., \& Tibayrenc, M. (2004). Lack of correlation between in vitro susceptibility to benznidazole and phylogenetic diversity of Trypanosoma cruzi, the agent of Chagas disease. Experimental Parasitology, 108, 24-31.

Volonté, M. G. \& Quiroga, P. (2013). Análisis farmacéutico, Universidad Nacional de La Plata. Buenos Aires: Edulp.

Wang, X., Beno-Movalem, D., Kobiler, I., Leikin-Frenkel, A., Lichter, A., \& Prusky, D. 2004. Expression of $\Delta 12$ fatty acid desaturase during the induced accumulation of the antifungal diene in avocado fruits. Molecular Plant Pathology, 5, 575-585.

Werner, A. B., \& Zulantay, I. (2011). Estado actual en el tratamiento de la enfermedad de Chagas. Revista Médica de Chile, 139, 247-257.

World Health Organization (WHO). 2015. Chagas disease (American trypanosomiasis). Fact sheet No340. Updated. March 2015. http://www.who.int/mediacentre/ factsheets/fs $340 / \mathrm{en} /$

Yasunaka, K., Abe, F., Nagayama, A., Okabe, H., LozadaPerez, L., Lopez-Villafranco, E., Estrada Muñiz, E., Aguilar, A., \& Reyes-Chilpa, R. (2005). Antibacterial activity of crude extracts from Mexican medicinal plants and purified coumarins and xanthones. Journal of Ethnopharmacology, 97, 293-299.

Yueqin, Z., Recio, M. C., Máñez, S., Giner, R. M., CerdáNicolás, M., \& Ríos, J. L. (2003). Isolation of two triterpenoids and a bioflavanone with anti-inflammatory activity from Schinus molle fruits. Planta Medica, 69, 893-898. 